\title{
Comment on the Paper "Condom-Assisted Transurethral Resection: A New Surgical Technique for Urethral Tumor", Surgical Science, Vol. 1, 2010, pp. 46-48*
}

\author{
Guven Aslan \\ Dokuz Eylul University, School of Medicine Department of Urology, Izmir, Turkey \\ E-mail: aslang@deu.edu.tr \\ Received December 21, 2010; revised April 11, 2011; accepted April 15, 2011.
}

\begin{abstract}
The authors introduce a new technical modification which facilitates endoscopic resection of urethra tumors located at navicular fossa by using a tipcut condom that covers the resectoscope and the penis. This technique can be used in the diagnosis and management of all kind male anterior urethral lesions at this location.
\end{abstract}

Keywords: Urethral Tumor, Transurethral Resection, Surgical Technique

I read with interest the article entitled "Condom-Assisted Transurethral Resection: A New Surgical Technique for Urethral Tumor" [1]. The authors introduce a new technical modification which facilitates endoscopic resection of urethra tumors located at navicular fossa. The case report will be of interest to surgeons experienced urethral lesions in their surgical practice.

Transitional cell carcinoma usually presents in the posterior urethra, mainly in the prostatic portion where the epithelium is made up of transitional cells and in continuity with the bladder epithelium [2]. Most carcinomas of the anterior urethra are squamous cell carcinoma [2]. Transitional cell carcinoma within the squamous lined navicular fossa is extremely rare. Numerous processes including foci of ectopic transitional epithelium, metaplasia and HPV infection have been proposed, and as yet, no evidence points to any one of these hypotheses $[3,4]$. Because limited number of cases have been reported and the scarcity of reports, treatment is not standard. Unlike squamous cell carcinoma of the anterior urethra, which usually requires surgical excision or partial penectomy, transitional cell carcinoma of this location can be managed with endoscopic local resection and fulguration thus preservation of the penis for noninvasive lesions.

*K. Sato, M. Suzuki, M. Nagata, H. Nishimatsu, A. Ishikawa, Y. Igawa H. Kume, Y. Homma, "Condom-Assisted Transurethral Resection: A New Surgical Technique for Urethral Tumor”, Surgical Science, Vol. 1 2010, pp. 46-48.
Many urologist find difficult endoscopic management of urethral tumors, especially tumors located at fossa navicularis. Because the location of urethral lesion placed is very close to urethral meatus, irrigant solution is usually spilled out making visualization poor. The authors devised a new practical surgical technique to facilitate TUR of urothelial tumors in fossa navicularis by using a tipcut condom that covers the resectoscope and the penis. The method described herein by the authors is not limited to the management of tumor recurrence after urothelial cancer surgery. This technique can be used in the diagnosis and management of all kind male anterior urethral lesions at this location.

I congratulate the authors for managing such a rare disease successfully and sharing their experience with us which could obviously be a significant contribution to the learning of the surgical fraternity.

\section{References}

[1] K. Sato, M. Suzuki, M. Nagata, H. Nishimatsu, A. Ishikawa, Y. Igawa, H. Kume and Y. Homma, "Condom-Assisted Transurethral Resection: A New Surgical Technique for Urethral Tumor”, Surgical Science, Vol. 1, 2010, pp. 46-48. doi:10.4236/ss.2010.12009

[2] R. L. Levine, “Urethral Cancer”, Cancer, Vol. 45, No. 7, 1980; pp. 1965-72.

[3] G. S Steele, J. R. Fielding, A. Renshaw, K. R. Loughlin, "Transitional Cell Carcinoma of the Fossa Navicularis", 
Urology, Vol. 50, 1997, pp. 792-795 doi:10.1016/S0090-4295(97)00318-X

[4] M. J. Resnick, A.J Wein, “Transitional Cell Carcinoma of the Fossa Navicularis in a Man with Preexisting Adenocarcinoma of the Prostate", Urologia Internationalis, Vol. 76, 2006, pp. 186-188. doi:10.1159/000090887 